\title{
Contrast sensitivity function and pituitary adenoma: a study of 40 cases
}

\author{
M Grochowicki, A Vighetto, S Berquet, G Sassolas
}

\begin{abstract}
The authors studied the contrast sensitivity function in $\mathbf{4 0}$ patients with pituitary adenoma. It seems to be a good index of compression of the visual pathway in this disease, but it lacks specificity and it cannot be used alone.
\end{abstract}

There are few studies using contrast sensitivity function (CS) in pituitary adenoma (PA) ${ }^{1-3}$ Usually associated with defects of the visual field, the alteration of CS may be total' or limited to low and middle spatial frequencies only. ${ }^{23} \mathrm{We}$ investigated such patients to determine whether loss of contrast sensitivity might be an early and sensitive index of compression of the visual pathway.

\section{Patients and methods}

We reviewed the CS of 40 patients aged from 15 to 48 years suffering from PA $(12$ microadenomas $<10 \mathrm{~mm} ; 28$ macroadenomas $\geqslant 10$ $\mathrm{mm})$. We used stationary vertical sinusoidal gratings on a television screen with a technique described elsewhere. ${ }^{4}$ Six spatial frequencies between 0.8 and 26 cycles per degree were presented. The mean of three determinations at each spatial frequency was compared with those of control subjects matched for age. Every value inferior to 2.5 standard deviation (SD) from the control population was considered to be pathological $(p<0 \cdot 01)$. The results were compared visual acuity (VA) data, visual field (VF) data (Goldmann kinetic perimetry and/or automatic static central perimetry performed on a Vision Monitor system), and with the degree of expansion of the tumour, as measured by CT scan and surgical findings.

Hôpital Neurologique et Neuro-Chirurgical Pierre Wertheimer, Consultation de NeuroOphtalmologie M Grochowicki A Vighetto $S$ Berquet

Service de Médecine Nucléaire Sassolas G

Correspondence to: $\operatorname{Dr} M$ Grochowicki, Consultation de Neuro-Ophtalmologie, Hôpital Neurologique et Neuro-Chirurgical Pierre Werro-Chirurgical Pierre Pinel, 69003 Lyon, France. Accepted for publication 4 January 1990

\section{Results}

The CS was altered in 21 out of 40 cases of pituitary adenoma. The patients could be separated into three groups with respect to CS:

Group I: normal CS $(\mathrm{n}=19)$. The VA was equal or superior to 20/25. Ten patients had intrasellar microadenomas; nine were macroadenomas with an extrasellar expansion but only one extended upwards, bulging the optic chiasm. The visual field was also normal in 13 cases but abnormal in 6 cases.

Group II: bilateral attenuation of CS $(n=13)$. The VA was decreased at least for one eye in 12 out of 13 cases (VA ranging from 0 to 20/25). The CS was not tested in one eye of four patients because of the poor VA. Attenuation of CS was observed in nine eyes in the high and middle frequency range, in eight eyes in the high frequency only, and was global in five eyes. The tumours were larger than in group I. Two were microadenomas and 11 were macroadenomas with suprasellar development. All these patients but one had a bitemporal hemianopia.

Group III: unilateral attenuation of CS $(\mathrm{n}=8)$. The VA was superior or equal to 20/30 in all eyes but one. Attenuation of CS was observed in the high and middle frequency range in three cases, in the high frequency range only in two cases, and was global in two cases. The CS was not tested in one eye because of the poor VA. All the tumours of group III were macroadenomas. The visual field was altered in five cases. In three patients whose CS was altered and visual field remained normal the tumour bulged upwards moderately through the diaphragma sellae.

\section{Discussion}

Loss of CS is usually associated with alteration of VA and visual field. In small intrasellar adenomas the CS, VA, and visual field are normal. Bilateral attenuation of CS corresponds to profound alteration of VA and bitemporal hemianopia frequently found with the largest tumours. Unilateral alteration of CS corresponds to miscellaneous conditions, and the CS may be altered even though the visual field remains normal. In three cases, attenuation of CS was observed without other signs. In these cases the CS may provide an early sign of upward development of a pituitary adenoma.

On the other hand, in group I the visual field was altered in six cases even though CS remained normal (three were microadenomas, three were macroadenomas). The discrepancies observed in these tests suggest that it is useful to compare CS and VF when investigating patients with pituitary adenomas.

In conclusion, $\mathrm{CS}$ seems to be a sensitive index of compression of the visual pathway in PA. However, alteration of this measurement alone is not diagnostic because of its lack of specificity. Its usefulness is limited to the follow-up in association with study of the visual field.

i Bodis-Wollner I, Diamond SP. The measurement of spatial contrast sensitivity in cases of blurred vision associated with cerebral lesions. Brain 1976; 99: 695-710.

2 Kupersmith MJ, Siegel IM, Carr RE. Subtle disturbances of vision with compressive lesions of the anterior visual pathway measured by contrast sensitivity. Ophthalmology 1982; 89: 68-72.

3 Lorance RW, Kaufman D, Wray SH, Mao C. Contrast visual testing in neurovisual diagnosis. Neurology $1987 ; 37$ : 923-9.

4 Vighetto A, Grochowicki M, Cousin J. Sensibilité au contraste spatial et sclérose en plaques. Rev Neurol (Paris). (In press). 